\title{
COHOMOLOGY OF QUASI-PROJECTIVE STIEFEL MANIFOLDS
}

\author{
A. DUANE RANDALL
}

1. Introduction. Let $V_{n, k}$ denote the Stiefel manifold of orthonormal $k$-frames in Euclidean $n$-space. The special orthogonal group $S O(2)$ acts freely on $V_{2 n, k}$ via the diagonal embedding of $S^{1}$ in $U(n)$ and the standard embedding of $U(n)$ into $S O(2 n)$ corresponding to realification $r: B U(n) \rightarrow B S O(2 n)$. The quasi-projective Stiefel manifold $P V_{2 n, k}$ is the quotient space of $V_{2 n, k}$ under this action of $S O(2)$. The spaces $P V_{2 n, k}$ are classifying spaces for sectioning multiples of a complex line bundle. If $X$ is a finite complex and $\xi$ a complex line bundle over $X$, then $n \xi$ has $k$ linearly independent real sections if and only if there is a map $f: X \rightarrow P V_{2 n, k}$ such that $f^{*} \eta_{0}=\xi$ where $\eta_{0}$ is the complex line bundle over $P V_{2 n, k}$ associated to the $S^{1}$-fibering $V_{2 n, k} \rightarrow P V_{2 n, k}$. In this paper we determine the cohomology algebras of the spaces $P V_{2 n, k}$.

2. Preliminaries. We first establish some notation. Let $R E\left(x_{i} \mid i \in I\right)$ denote the exterior algebra over a ring $R$ with generators $x_{i}$ of degree $i$. Let $V\left(x_{1}, \cdots, x_{m}\right)$ denote the commutative associative algebra over $Z_{2}$ on generators $x_{1}, \cdots, x_{m}$ such that the monomials $x_{1} \epsilon_{1} \cdots x_{m}{ }^{\epsilon_{m}}$ with $\epsilon_{i}=0$ or 1 form an additive basis. Let $\left\{{ }_{p} E_{r}(X)\right\}$ denote the $\bmod p$ Bockstein spectral sequence for $X$ with ${ }_{p} E_{1}(X)=H^{*}\left(X ; Z_{p}\right)$. $C_{r, i}$ denotes the binomial coefficient $\left(\begin{array}{c}r \\ i\end{array}\right)$. Let $\rho_{p}$ denote the universal coefficient map $H^{*}(; Z) \rightarrow H^{*}\left(; Z_{p}\right)$ for any prime $p$ and let $\rho_{0}$ denote the map $H^{*}(; Z) \rightarrow H^{*}(; Q)$. Denote the image of an integral class $x$ under the projection $H^{*}(X ; Z) \rightarrow H^{*}(X ; Z) /$ Tors by $\bar{x}$. Finally let $J_{n, k}$ represent the set of all integers $j$ such that $[(2 n-k) / 2]<j<n$ where $0<k<2 n$. We write $H^{*}\left(C P^{\infty}\right)=Z[\beta]$.

Recall from [5] the cellular structure of the Stiefel manifold $V_{2 n, k}$ obtained from an embedding of real projective space $R P^{2 n-1}$ into $O(2 n)$ composed with the projection map $O(2 n) \rightarrow V_{2 n, k}$. The image of $R P^{2 j}$ determines a class $P^{2 j}$ in $H^{2 j}\left(V_{2 n, k} ; Z\right)$ of order 2 for every $j \in J_{n, k}$. Set $x_{2 j}=\rho_{2}\left(P^{2 j}\right)$. $R P^{2 n-k}$ determines a free integral class $y_{2 n-k}$ for $k$ even. Let $x_{2 n-k}=\rho_{2}\left(y_{2 n-k}\right)$ for $k$ even and let $x_{2 n-k}$ be the unique class such that $\mathrm{Sq}^{1} x_{2 n-k}=x_{2 n-k+1}$ for $k$ odd. By [2]

Received by the editors November 18, 1971.

AMS (MOS) subject classifications (1970). Primary 55F40; Secondary 55F25, $55 \mathrm{H} 10$. 
there is a class $y_{2 n-1} \in H^{2 n-1}\left(V_{2 n, k}\right)$ such that $\tau\left(y_{2 n-1}\right)=\chi_{2 n}$ where $\tau$ denotes transgression in the spectral sequence for the fibration

$$
V_{2 n, k} \rightarrow B S O(2 n-k) \rightarrow B S O(2 n)
$$

and $\chi_{2 n}$ is the Euler class. Set $x_{2 n-1}=\rho_{2}\left(y_{2 n-1}\right)$. By [1] and [5]

$$
\begin{aligned}
H^{*}\left(V_{2 n, k} ; Z_{2}\right) & =V\left(x_{2 n-k}, \cdots, x_{2 n-1}\right) \text { and } \\
\mathrm{Sq}^{i} x_{j} & =C_{j, i} x_{i+j} .
\end{aligned}
$$

For every $j \in J_{n, k}$, there is a class $y_{4 j-1}$ in $H^{4 j-1}\left(V_{2 n, k}\right)$ such that $\tau\left(2 y_{4 j-1}\right)=$ the Pontryagin class $p_{j}$ and $\rho_{2}\left(y_{4 j-1}\right)=x_{2 j} x_{2 j-1}+x_{4 j-1}$ from $[2,30.10]$. By [1]

$$
H^{*}\left(V_{2 n, k}\right) / \text { Torsion }=Z E\left(\bar{y}_{2 n-k}, \bar{y}_{2 n-1}, \bar{y}_{4 j-1} \mid j \in J_{n, k}\right)
$$

where $\bar{y}_{2 n-k}$ is omitted for $k$ odd. For integers $s, t \in J_{n, k}$ with $s<t$ let $u_{s, t}$ be the integral class of order 2 such that $\rho_{2}\left(u_{s, t}\right)=x_{2 s} x_{2 t-1}+$ $x_{2 t} x_{2 s-1}$. We state the following known

Proposition 2.3. The classes $y_{2 n-1}, y_{4 j-1}, u_{s, t}, P^{2 j}$ for $j, s, t \in J_{n, k}$ with $s<t, y_{2 n-k}$ for $k$ even, and the unit generate the algebra $H^{*}\left(V_{2 n, k}\right)$.

Consider the following commutative diagram of fibrations.

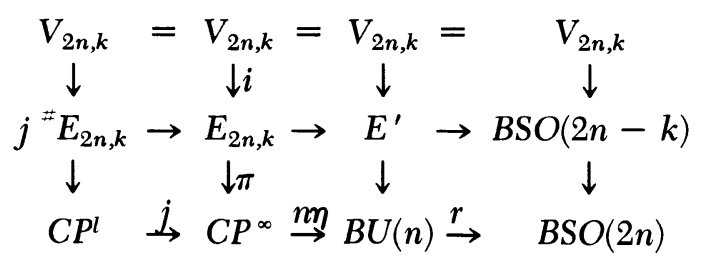

Here $\pi: E_{2 n, k} \rightarrow C P^{\infty}$ is the principal fibration induced from the fibration $B S O(2 n-k) \rightarrow B S O(2 n)$ by the map $r \circ n \eta: C P^{\infty} \rightarrow$ $B S O(2 n)$ classifying the $n$-fold sum of the Hopf bundle $\eta$ over $C P^{\infty}$ regarded as a real vector bundle. By construction $E_{2 n, k}$ is the classifying space for finding $k$ independent real sections to the $n$-fold Whitney sum of a complex line bundle over a finite complex. The method of proof of [3, Proposition 1.3] yields the following

Proposition 2.5. The spaces $P V_{2 n, k}$ and $E_{2 n, k}$ have the same homotopy type.

Consider the following homotopy commutative diagram of vertical fibrations with $m=n-k$. 


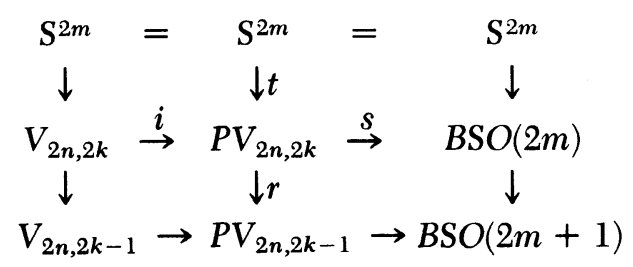

The fibration $S^{2 m} \rightarrow P V_{2 n, 2 k} \rightarrow P V_{2 n, 2 k-1}$ is totally nonhomologous to zero so we obtain

Proposition 2.7. $H^{*}\left(P V_{2 n, 2 k}\right)=H^{*}\left(P V_{2 n, 2 k-1}\right) \otimes H^{*}\left(S^{2 m}\right)$ as $H^{*}\left(P V_{2 n, 2 k-1}\right)$-modules.

We select $X$ uniquely in $H^{2 m}\left(P V_{2 n, 2 k}\right)$ such that $t^{*} X$ generates $\bar{H}^{*}\left(\mathrm{~S}^{2 m}\right)$ and

$$
s^{*} \chi_{2 m}=2 X+C_{n, k} \omega^{m}
$$

where $\omega$ denotes $\pi^{*} \beta$ for $\pi: P V_{2 n, 2 k} \rightarrow C P^{\infty}$ in (2.4). This selection is possible by [6, Theorem A] using the natural map $Y_{n, k} \rightarrow P V_{2 n, 2 k}$ where $Y_{n, k}$ denotes the complex projective Stiefel manifold. Since $\chi_{2 m}^{2}=p_{m}$ in $H^{*}(B S O(2 m))$, it follows that

$$
4 X^{2}=C_{n, k}\left(1-C_{n, k}\right) \omega^{2 m}-4 C_{n, k} X \omega^{m} .
$$

Thus we consider the spaces $P V_{2 n, k}$ primarily for $k$ odd.

We remark that the problem of determining the geometric dimension of $m \eta$ based on $C P^{l}$ is equivalent to finding the largest integer $k$ such that $j^{\#} E_{2 n, k} \rightarrow C P^{l}$ has a section where $j^{*} E_{2 n, k} \rightarrow C P^{l}$ is the fibration in (2.4) induced from $\pi$ via the standard embedding $j: C P^{l} \rightarrow$ $C P^{\infty}$. (See [7] for the case $l=n-1$.) Note that $P V_{2 n, 1}$ is $C P^{n-1}$.

3. Rational and $\bmod p$ cohomology of $P V_{2 n, k}$. Let $F$ denote $Q$ or $Z_{p}$ for an odd prime $p$. From (2.2) and (2.3) $H^{*}\left(V_{2 n, k} ; F\right)=$ $F E\left(\tilde{y}_{2 n-k}, \tilde{y}_{2 n-1}, \tilde{y}_{4 j-1} \mid j \in J_{n, k}\right)$ where $\tilde{y}_{2 n-k}$ is omitted for $k$ odd, and denotes the image under $\rho: H^{*}(; Z) \rightarrow H^{*}(; F)$.

THEOREM 3.1. Let $k$ be an odd integer with $k<n+1$. Then $H^{*}\left(P V_{2 n, k} ; F\right)=F[\tilde{\omega}] /\left(\tilde{\boldsymbol{\omega}}^{n}\right) \otimes F E\left(v_{4 j-1} \mid j \in J_{n, k}\right)$ where $i^{*} v_{p}=\tilde{y}_{p}$ and $\omega=\pi^{*} \beta$.

Proof. The Serre spectral sequence for the fibration $V_{2 n, k} \stackrel{i}{\rightarrow} P V_{2 n, k}$ $\stackrel{\pi}{\rightarrow} C P^{\infty}$ in $(2.4)$ with coefficients $F$ has $E_{2}{ }^{*, *}=F[\tilde{\beta}] \otimes$ $F E\left(\tilde{y}_{2 n-1}, \tilde{y}_{4 j-1} \mid j \in J_{n, k}\right)$. Since $\tau\left(2 y_{4 j-1}\right)=p_{j}(n \eta)=C_{n, j} \beta^{2 j}$ in the integral spectral sequence for $\pi$, the fiber is transgressively generated over $F$. By dimensionality $d_{2 n}$ is the first nonzero differential and $d_{2 n}\left(\tilde{y}_{2 n-1}\right)=\tau\left(\tilde{y}_{2 n-1}\right)=\tilde{\chi}(n \eta)=\tilde{\boldsymbol{\beta}}^{n}$. Note that the image of the ideal 
$\left(\tilde{\boldsymbol{\beta}}^{n}\right) \quad$ in $\quad E_{2 n^{*}+1}^{* *} \quad$ is $\quad 0$ and $E_{2 n^{*}+1}^{* *}=E_{2 n^{*}, 1}^{*, 0} \otimes E_{2 n+1}^{0, *}$. All the following differentials are trivial so $E_{\infty}^{* * *}=E_{2 n+1}^{* * *}$. The result follows from [1, Proposition 8.1] .

Let $l$ denote the smallest integer in $J_{n, k}$. Given an odd prime $p$, let $N(p)$ denote the smallest integer $j$ in $J_{n, k}$ such that $p$ does not divide $C_{n, j}$. If no such integer $j$ exists, set $N(p)=\infty$.

ThEOREM 3.2. Let $k$ be an odd integer with $k>n$. If $n \neq 2 l$

$$
H^{*}\left(P V_{2 n, k} ; Q\right)=Q[\tilde{\omega}] /\left(\tilde{\omega}^{2 l}\right) \otimes Q E\left(v_{2 n-1}, v_{4 j-1} \mid l<j<n\right)
$$

with $i^{*} v_{s}=\tilde{y}_{s}=\rho_{0}\left(y_{s}\right)$. If $n=2 l$,

$$
H^{*}\left(P V_{2 n, k} ; Q\right)=Q[\tilde{\omega}] /\left(\tilde{\omega}^{n}\right) \otimes Q E\left(v_{4 j-1} \mid j \in J_{n, k}\right)
$$

where $i^{*} v_{4 l-1}=2 \tilde{y}_{4 l-1}-C_{n, l} \tilde{y}_{2 n-1}$ and $i^{*} v_{s}=\tilde{y}_{s}$ otherwise. If $2 N(p)<n$,

$$
\begin{aligned}
H^{*}\left(P V_{2 n, k} ; Z_{p}\right)= & Z_{p}[\tilde{\omega}] /\left(\tilde{\omega}^{2 N(p)}\right) \\
& \otimes Z_{p} E\left(v_{2 n-1}, v_{4 j-1} \mid j \in J_{n, k}, j \neq N(p)\right)
\end{aligned}
$$

with $i^{*} v_{s}=\tilde{y}_{s}=\rho_{p}\left(y_{s}\right)$. If $2 N(p)>n$,

$$
H^{*}\left(P V_{2 n, k} ; Z_{p}\right)=Z_{p}[\tilde{\omega}] /\left(\tilde{\omega}^{n}\right) \otimes Z_{p} E\left(v_{4 j-1} \mid j \in J_{n, k}\right)
$$

with $i^{*} v_{s}=\tilde{y}_{s}$. If $2 N(p)=n$,

$$
H^{*}\left(P V_{2 n, k} ; Z_{p}\right)=Z_{p}[\tilde{\omega}] /\left(\tilde{\omega}^{n}\right) \otimes Z_{p} E\left(v_{4 j-1} \mid j \in J_{n, k}\right)
$$

where $i^{*} v_{4 N(p)-1}=2 \tilde{y}_{4 N(p)-1}-C_{n, N(p)} \tilde{y}_{2 n-1}$.

Theorem 3.2 follows similarly from the proof of (3.1). From (3.2) and (2.7) we obtain the following

Corollary 3.3. $H^{*}\left(P V_{2 n, k}\right)$ has $p$-torsion for an odd prime $p$ if and only if $k>n+2$ for $n$ even, $k>n+1$ for $n$ odd, and $p$ divides $C_{n, l}$.

The $Z_{2}$ cohomology algebra of $P V_{2 n, k}$ and module structure over the Steenrod algebra $A$ have essentially been determined up to a small indeterminacy by Gitler and Handel in [3]. Let $N$ denote the smallest integer $j$ with $C_{n, j}$ odd and $2 n-k+1 \leqq 2 j \leqq 2 n$. Applying the proof of $[3$, Theorem 2.8] gives the following

THEOREM 3.4. As an algebra

$$
\begin{aligned}
H^{*}\left(P V_{2 n, k} ; Z_{2}\right)= & Z_{2}[\alpha] /\left(\alpha^{N}\right) \\
& \otimes V\left(z_{2 n-k}, \cdots, z_{2 N-2}, z_{2 N}, \cdots, z_{2 n-1}\right)
\end{aligned}
$$


where $i^{*} z_{p}=x_{p}$ and $\alpha=\rho_{2}(\omega)$. If $C_{2 n, q}$ is even,

$$
\mathrm{Sq}^{i} z_{q-1}=\sum_{k \in K} C_{q-1-2 k, i-2 k} w_{2 k}\left(n \eta_{0}\right) z_{q+i-2 k-1}+\lambda(q, i) \text {. }
$$

If $C_{2 n, q}$ is odd, then $q=2 s$ and

$$
\begin{aligned}
S q^{i} z_{q-1}= & \sum_{k \in K} C_{q-1-2 k, i-2 k} w_{2 k}\left(n \eta_{0}\right) z_{q+i-2 k-1} \\
& +\sum_{j, k \in J} C_{2 N-1-2 k, j-2 k} \operatorname{Sq}^{i-j} \alpha^{s-N} w_{2 k}\left(n \eta_{0}\right) z_{2 N+j-2 k-1} .
\end{aligned}
$$

Here $\lambda(q, i)=0$ if $q+i$ is even, and $\lambda(q, i)=\epsilon \alpha^{r}$ if $q+i-1=2 r$ where $\epsilon=0$ or 1 . $K=\{k \mid 0 \leqq 2 k \leqq i$ and $q+i-2 k \neq 2 N\}$ and $J=\{j, k \mid 0 \leqq 2 k<j \leqq i\}$.

Suppose now that $k<n+2$ for $n$ odd and $k<n+3$ for $n$ even. We shall show that all torsion in $H^{*}\left(P V_{2 n, k}\right)$ has order 2. Note from (3.4) that $\mathrm{Sq}^{1} z_{2 j-1}=z_{2 j}+\lambda(2 j, 1)$ where $j<N$ and $2 n-k<2 j$. If $\lambda(2 j, 1) \neq 0$, we define $z_{2 j}$ to be Sq ${ }^{1} z_{2 j-1}$. Take $s \in J_{n, k}$ with $s \neq N$. If $s<N$, define

$$
Z_{4 s-1}=z_{2 s-1} z_{2 s}+\sum_{\substack{j=2 s-n+1 \\ j \neq 2 s-N}}^{s} C_{n, j} \alpha^{j} z_{4 s-2 j-1}+\lambda_{s} \alpha^{2 s-n} z_{2 n-1}
$$

where

$$
\lambda_{s}=C_{n, 2 s-N}+\sum_{j=2 s-n+1}^{2 s-N-1} C_{n, j} C_{n, 2 s-j} \in Z_{2}
$$

If $s>N$, define

$$
\begin{aligned}
Z_{4 s-1}= & z_{2 s-1} z_{2 s}+\sum_{j=2 s-n+1}^{N-1} C_{n, j} \alpha^{j} z_{4 s-2 j-1} \\
& +C_{n, s} \alpha^{s-N} z_{2 N} z_{2 s-1} \\
& +C_{n, s} \sum_{l=2 N-n+1}^{3 N-2 s-1} C_{n, l} \alpha^{2 s-2 n+l} z_{4 N-2 l-1}
\end{aligned}
$$

Note from (3.4) that $Z_{4 s-1} \in \operatorname{ker~Sq}{ }^{1}$, and $Z_{4 s-1}$ is not in im Sq ${ }^{1}$ since $i^{*} Z_{4 s-1}=x_{2 s-1} x_{2 s}$. Clearly $Z_{4 s-1}^{2}$ is in im Sq ${ }^{1}$. Note also that $\mathrm{Sq}^{1} z_{2 n-1}=\alpha^{n-N_{z_{2 N}}}$ and, for $0 \leqq j<n-N$, 


$$
\left(\alpha^{j} z_{2 N}\right)^{2}=\operatorname{Sq}^{1}\left(\alpha^{2 j} \sum_{l=2 N-n+1}^{N-1} \alpha^{l} C_{n, l} z_{4 N-2 l-1}\right) .
$$

Let $T$ denote the graded algebra over $Z_{2}$ with trivial multiplication on generators $z_{2 N}, z_{2 N} z_{2 n-1}$, and $\alpha^{2 N-n} z_{2 n-1}$. Similar computation using (3.4) yields the following for $k$ odd and $k<n+2$.

Proposition 3.5.

$$
\begin{aligned}
{ }_{2} E_{2}\left(P V_{2 n, k}\right)= & Z_{2}[\alpha] /\left(\alpha^{N}\right) \\
& \otimes Z_{2} E\left(Z_{4 s-1} \mid s \in J_{n, k}, s \neq N\right) \otimes T / 1
\end{aligned}
$$

where $I$ is the ideal generated by $\alpha^{n-N} \otimes 1 \otimes z_{2 N}$ and $\alpha^{n-N} \otimes 1 \otimes$ $\alpha^{2 N-n} z_{2 n-1}$.

Corollary 3.6. All torsion in $H^{*}\left(P V_{2 n, k}\right)$ has order 2 where $k<n+2$ for $n$ odd and $k<n+3$ for $n$ even.

Proof. Assume $k$ is odd and $k<n+2$. To show ${ }_{2} E_{2}\left(P V_{2 n, k}\right)=$ ${ }_{2} E_{\infty}\left(P V_{2 n, k}\right)$, it suffices to define an isomorphism $\varphi:{ }_{2} E_{2}\left(P V_{2 n, k}\right) \rightarrow$ $H^{*}\left(P V_{2 n, k} ; Q\right)$ of graded vector spaces over $Z_{2}$. Define $\varphi\left(\alpha^{s}\right)=\tilde{\omega}^{s}$ for $s<N, \varphi\left(\alpha^{s} z_{2 N}\right)=\tilde{\omega}^{N+s}$ and $\varphi\left(\alpha^{s+2 N-n} z_{2 n-1}\right)=\tilde{\omega}^{s} v_{4 N-1}$ for $0 \leqq$ $s<n-N, \varphi\left(\alpha^{s} z_{2 N} z_{2 n-1}\right)=\tilde{\omega}^{s+n-N} v_{4 N-1}$ for $0 \leqq s<N$, and $\varphi\left(Z_{4 j-1}\right)$ $=v_{4 j-1}$ for $j \in J_{n, k}$ and $j \neq N$. Extend $\varphi$ to an isomorphism and apply (2.7) for $k$ even.

\section{Integral cohomology.}

Case I. We assume in Case I that $k<n+1$ with $k$ odd. We determine the differentials and $E_{\infty}^{* *}$ for the integral spectral sequence for the fibration $V_{2 n, k} \stackrel{i}{\rightarrow} P V_{2 n, k} \rightarrow C P^{\infty}$ in (2.4) and then use the Gysin sequence to specify generators for $H^{*}\left(P V_{2 n, k}\right) . \quad E_{2}{ }^{*}, *=Z[\beta] \otimes$ $H^{*}\left(V_{2 n, k}\right)$. Since $\tau\left(P^{2 j}\right)=\delta w_{2 j}$ for the fibration $B S O(2 n-k) \rightarrow$ $B S O(2 n)$ where $\delta$ denotes the integral Bockstein operator, $P^{2 j}$ for $j \in J_{n, k}$ survives in the integral spectral sequence for $\pi$. $\tau\left(y_{2 n-1}\right)=$ $\chi(n \eta)=\beta^{n}$ so $E_{2 n+1}^{0,0}=0$ for $p>2 n$. All differentials kill $2 y_{4 j-1}$ for $j \in J_{n, k}$ since $\tau\left(2 y_{4 j-1}\right)=p_{j}(n \eta)$. Note that $d_{2 N}$ is the first nontrivial differential in the integral spectral sequence for $\pi$ since $d_{2 N}$ is the first nontrivial differential in the $Z_{2}$ spectral sequence by (3.4) and $d_{2 n}$ is the only nontrivial differential with $F$ coefficients by (3.1). If $N=n$, clearly

$$
E_{\infty}^{*, *}=E_{2 n+1}^{*, *}=Z[\beta] /\left(\beta^{n}\right) \otimes H^{*}\left(V_{2 n, k}\right) /\left(y_{2 n-1}\right) .
$$

Assume $N<n$. Now $d_{2 N}\left(1 \otimes y_{4 N-1}\right)=\beta^{N} \otimes P^{2 N}$ since

$$
d_{2 N}\left(1 \otimes x_{2 N-1} x_{2 N}\right)=\beta^{N} \otimes x_{2 N}=\rho_{2}\left(\beta^{N} \otimes P^{2 N}\right) .
$$


Similarly, $\quad d_{2 N}(1 \otimes u)=\beta^{N} \otimes P^{2 j} \quad$ where $\quad u=u_{j, N} \quad$ if $j<N$, and $u=u_{N, j}$ if $N<j$. Since $d_{2 j}\left(y_{4 j-1}\right)=$ image of $c_{j} \otimes P^{2 j}$ in $E_{2 j}^{* *}$ in the integral spectral sequence for $E^{\prime} \rightarrow B U(n)$ in (2.4), one checks that $d_{2 j}\left(y_{4 j-1}\right)=$ image of $C_{n, j} \beta^{j} \otimes P^{2 j}$ in $E_{2 j}^{* *}$ in the integral spectral sequence for $\pi$, and $d_{2 r}\left(y_{4 j-1}\right)=0$ for $j<r<2 j$ if $d_{2 j}\left(y_{4 j-1}\right)=0$. Note that for $s, t \in J_{n, k}-\{N\}$ with $s<t$,

$$
u_{s, t}=i^{*} U_{s, t}
$$

where $U_{s, t}=\delta\left(z_{2 s-1} z_{2 t-1}\right)$ since

$$
\begin{aligned}
\mathrm{Sq}^{1}\left(z_{2 s-1} z_{2 t-1}\right)= & z_{2 s} z_{2 t-1}+z_{2 s-1} z_{2 t} \\
& +C_{n, s} \alpha^{s-N} z_{2 N} z_{2 t-1}+C_{n, t} \alpha^{t-N} z_{2 N} z_{2 s-1}
\end{aligned}
$$

by (3.4). Thus $E_{2 N+1}^{*, *}=Z[\beta] \otimes E_{2 N+1}^{0, *} / K$ where $K$ is the ideal generated by $\beta^{N} \otimes P^{2 j}$ for $j \in J_{n, k}$. The differentials $d_{p}$ are trivial for $p>2 N$ and $p \neq 2 n$ so it follows that

$$
E_{\infty}^{* * *}=E_{2 n+1}^{*, *}=Z[\beta] /\left(\beta^{n}\right) \otimes E_{2 n+1}^{0, *} / K
$$

as graded algebras.

The only nontrivial extension from $E_{\infty}^{* *}$ to $H^{*}\left(P V_{2 n, k}\right)$ is the nontrivial extension of $Z$ by $Z_{2}$. Since $\tau\left(z_{2 N-1}\right)=\rho_{2}\left(\beta^{N}\right)$, it follows from the universal example for division by 2 that

$$
\omega^{N}=\pi^{*} \beta^{N}=2 e_{2 N} \quad \text { with } i^{*} e_{2 N}=P^{2 N} \text { and } \rho_{2}\left(e_{2 N}\right)=z_{2 N} .
$$

Consider the following commutative diagram.

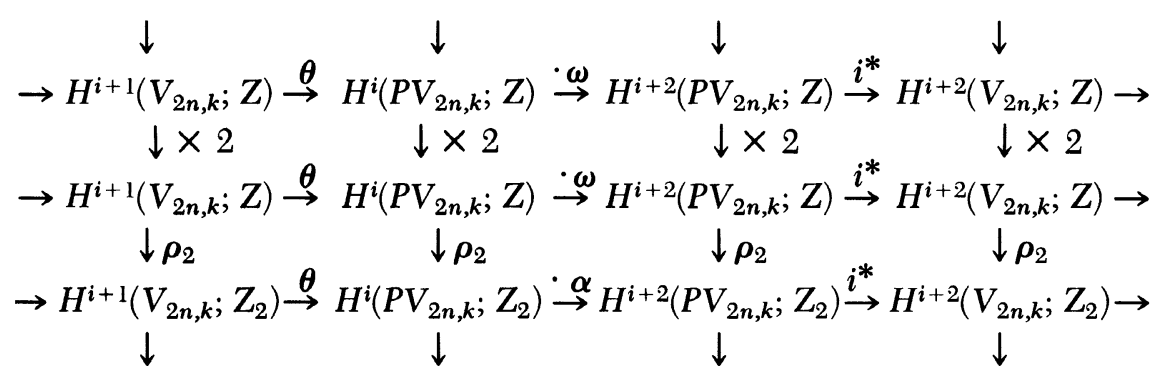

The above rows are the Gysin sequence for the fibration $S^{1} \rightarrow V_{2 n, k}$ $\rightarrow P V_{2 n, k}$.

For $s \in J_{n, k}-\{N\}$, we define

$$
Y_{2 s}=\delta\left(z_{2 s-1}\right)
$$


Note that $i^{*} Y_{2 s}=P^{2 s}$ and $\rho_{2}\left(Y_{2 s}\right)=z_{2 s}$. We define

$$
V_{s}=\delta\left(z_{2 s-1} z_{2 n-1}\right) \in H^{2 n+2 s-1}\left(P V_{2 n, k)}\right. \text {. }
$$

Clearly $\quad 2 V_{s}=0 \quad$ and $\quad \rho_{2}\left(V_{s}\right)=z_{2 s} z_{2 n-1}+\alpha^{n-N} z_{2 N} z_{2 s-1}+$

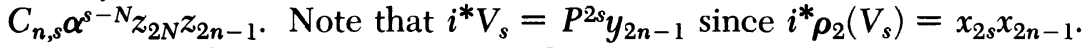
For any $j \in J_{n, k}-\{N\}$, we now show there exists (4.7) $X_{4 j-1} \in H^{4 j-1}\left(P V_{2 n, k}\right)$ with $i^{*} X_{4 j-1}=y_{4 j-1}$ and $\rho_{2}\left(X_{4 j-1}\right)=Z_{4 j-1}$. Let $u$ be any class in $H^{4 j-1}\left(P V_{2 n, k}\right)$ with $i^{*} u=y_{4 j-1}$. Then $\rho_{2}(u)=$ $Z_{4 j-1}+\alpha z$ with $z \in H^{4 j-3}\left(P V_{2 n, k} ; Z_{2}\right)$ by (4.4). Sq ${ }^{1}(\alpha z)=0$ so $\alpha z=$ $\rho_{2}(Z)$, and $i^{*} Z=2 V$ since $\rho_{2}\left(i^{*} Z\right)=i^{*}\left(\rho_{2} Z\right)=i^{*}(\alpha z)=0$. Select $Z^{\prime}$ such that $i^{*} Z^{\prime}=V$ by (4.2). Then $Z-2 Z^{\prime}=\omega Z^{\prime}$ and $X_{4 j-1}=u+$ $\omega Z^{\prime}$ satisfies (4.7).

Take any class $u \in H^{4 N-1}\left(P V_{2 n, k}\right)$ with $i^{*} u=2 y_{4 N-1}$. Then $\rho_{2}(u) \in \operatorname{kerSq}^{1} \cap \operatorname{ker} i^{*}$ so $\rho_{2}(u)=\alpha w$ with $\mathrm{Sq}^{1} w \neq 0$ by (4.4). It follows that $\rho_{2}(u)=\alpha^{2 N-n} z_{2 n-1}+\alpha \rho_{2}(V)$ for $V \in H^{4 N-3}\left(P V_{2 n, k}\right)$. Define $X_{4 N-1}=u+\omega V$ and note that

$$
i^{*} X_{4 N-1}=2 y_{4 N-1} \text { and } \rho_{2}\left(X_{4 N-1}\right)=\alpha^{2 N-n} z_{2 n-1} .
$$

Similarly, it follows from (4.4) and the fibration $V_{2 N-1, k+2 N-2 n-1} \rightarrow$ $P V_{2 n, k} \rightarrow P V_{2 n, 2 n-2 N+1}$ that we can choose $Y \in H^{2 n+2 N-1}\left(P V_{2 n, k}\right)$ so that

$$
2 Y=\omega^{n-N} X_{4 N-1}, \quad i^{*} Y=P^{2 N} y_{2 n-1}, \quad \text { and } \quad \rho_{2}(Y)=z_{2 N} z_{2 n-1} .
$$

One checks using (4.4) that

$$
\omega^{2 N-n} Y=e_{2 N} X_{4 N-1} .
$$

Note that $H^{*}\left(P V_{2 n, k} ; Q\right)=Q[\tilde{\omega}] /(\tilde{\boldsymbol{\omega}}) \otimes Q E\left(\tilde{X}_{4 j-1} \mid j \in J_{n, k}\right)$. In summary we have the following

THEOREM 4.11. Suppose that $k<n+1$ with $k$ odd. If $N=n$, $H^{*}\left(P V_{2 n, k}\right)=H^{*}\left(C P^{n-1}\right) \otimes H^{*}\left(V_{2 n, k}\right) /\left(y_{2 n-1}\right)$ as algebras. If $N<n$, $H^{*}\left(P V_{2 n, k}\right)$ is generated by the classes $\omega, e_{2 N}, Y_{2 s}, V_{s}, X_{4 j-1}, U_{s, t}$ and $Y$ where $j \in J_{n, k}$ and $s, t \in J_{n, k}-\{N\}$ with $s<t$. Relations among the generators and the product structure are determined by the rational and $\mathrm{Z}_{2}$ cup products.

REMark. $H^{*}\left(P V_{2 n, k}\right)$ contains the subalgebra $Z[\omega] /\left(\omega^{n}\right) \otimes$ $Z E\left(X_{4 j-1} \mid j \in J_{n, k}\right)$.

Case II. We assume that $n$ is even and $k=n+1$. Thus $n=2 l$. Set $d_{l}=\frac{1}{2} C_{n, l}$. Choose $X_{4 l-1}$ in $H^{2 n-1}\left(P V_{2 n, k}\right)$ such that

$$
i^{*} X_{4 l-1}=y_{4 l-1}-d_{l} y_{2 n-1} \text { and } \rho_{2}\left(X_{4 l-1}\right)=Z_{4 l-1} \text {. }
$$


Then $H^{*}\left(P V_{2 n, k}\right)$ is again given by (4.11).

\section{Integral cohomology.}

Case III. Finally we assume $2 l<n$ with $k$ odd. Let $d_{j}$ denote $\frac{1}{2} C_{n, j}$ for $l \leqq j<N$. Set $b_{l}=d_{l}$ and inductively define $b_{i}=$ G.C.D. $\left(d_{i}, b_{i-1}\right)$ for $l<i<N$.

Set $b_{N}=$ G.C.D. $\left(b_{N-1}, C_{n, N}\right)$. If $N=l$, set $b_{N}=C_{n, l}$. Define $b_{j}$ inductively for $2 N \leqq 2 j<n$. Suppose $b_{j}>1$. Set $b_{j+1}=$ G.C.D. $\left(b_{j}, \lambda_{j}\right)$ where $\lambda_{j} \in Z_{b_{j}}$ is chosen uniquely such that $C_{n, j+1}=2 \lambda_{j} \bmod b_{j}$. If $b_{j}=1$, set $b_{i}=1$ for $2 j<2 i<n$. The argument of [6, Proposition 5] shows

Proposition 5.1. Ker $\pi^{*}=\left[b_{l} \beta^{2 l}, \cdots, b_{j} \beta^{2 j}, \cdots, \beta^{n}\right]$ for $2 l \leqq 2 j<n$. $\operatorname{Ker} \bar{\pi}^{*}=\left[b_{l} \beta^{2 l+1}, \cdots, b_{j} \beta^{2 j+1}, \cdots, \beta^{n+1}\right]$ where $\overline{\bar{\pi}}:\left(P V_{2 n, k}, V_{2 n, k}\right)$ $\rightarrow\left(C P^{\infty}, *\right)$.

Set $a_{i}=b_{i-1} / b_{i}$ for $2 l<2 i<n$. Set $y_{4 N-1}=2 y_{4 N-1}$. Recall $T^{q-1} / \mathrm{im}^{*}=\operatorname{Ker}^{q} \pi^{*} / \operatorname{Ker}^{q} \bar{\pi}^{*}$ where $T^{q-1} \subseteq H^{q-1}\left(V_{2 n, k}\right)$ denotes the subgroup of transgressive elements. Thus we obtain from (5.1) the following

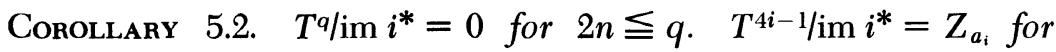

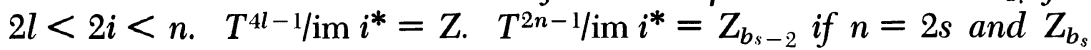
if $n=2 s+1$.

Thus there exist classes $X_{4 j-1}$ in $H^{4 j-1}\left(P V_{2 n, k}\right)$ for $2 l<2 j<n$ such that $i^{*} X_{4 j-1}=a_{j} y_{4 j-1}$. If $n=2 s+1$, choose $X_{2 n-1}$ in $H^{2 n-1}\left(P V_{2 n, k}\right)$ such that $i^{*} X_{2 n-1}=b_{s} y_{2 n-1}$. If $n=2 s$, choose $X_{2 n-1}$ such that $i^{*} X_{2 n-1}=b_{s-1} y_{2 n-1}$, and define $X_{4 s-1}$ so that $i^{*} X_{4 s-1}=y_{4 s-1}-$ $d_{s} y_{2 n-1}$ if $s<N$ and $i^{*} X_{4 s-1}=y_{4 s-1}-\lambda_{s} y_{2 n-1}$ if $s>N$. Select $X_{4 j-1}$ so that $i^{*} X_{4 j-1}=y_{4 j-1}$ for $n<2 j<2 n$. Choose a fixed set of the above classes arbitrarily. Let $p$ be a fixed odd prime and set $I_{p}=$ $\left\{j|2 l<2 j<n, p| a_{j}\right\} \cup\{l\}$. For $j \in I_{p}$ with $j \neq N(p)$, set $\bar{v}_{4 j-1}=$ $v_{4 j-1}$ from (3.2). Define $\bar{v}_{4 j-1}=\rho_{p}\left(X_{4 j-1}\right)$ for $l<j<n, j \notin I_{p}$, $j \neq N(p)$. Set $\bar{v}_{2 n-1}=\rho_{p}\left(X_{2 n-1}\right)$ if $p \nmid b_{s-1}$. Then

$$
\begin{aligned}
H^{*}\left(P V_{2 n, k} ; Z_{p}\right)= & Z_{p}[\tilde{\omega}] /\left(\tilde{\boldsymbol{\omega}}^{2 N(p)}\right) \\
& \otimes Z_{p} E\left(\bar{v}_{4 j-1}, \bar{v}_{2 n-1} \mid j \in J_{n, k}, j \neq N(p)\right), \\
= & Z_{p}[\tilde{\omega}] /\left(\tilde{\boldsymbol{\omega}}^{n}\right) \otimes Z_{p} E\left(\bar{v}_{4 j-1} \mid j \in J_{n, k}\right), \\
& \text { if } 2 N(p)<n,
\end{aligned}
$$

Note that 


$$
H^{*}\left(P V_{2 n, k} ; Q\right)=Q[\tilde{\boldsymbol{\omega}}] /\left(\tilde{\boldsymbol{\omega}}^{2 l}\right) \otimes Q E\left(\tilde{\boldsymbol{v}}_{4 j-1}, \tilde{v}_{2 n-1} \mid l<j<n\right)
$$

where $\tilde{v}_{4 j-1}=\rho_{0}\left(X_{4 j-1}\right)$ and $\tilde{v}_{2 n-1}=\rho_{0}\left(X_{2 n-1}\right)$. Arrange $I_{p}$ so that $l=i(0)<i(1)<\cdots<i(j)<\cdots<i(t)$ and write $b_{i(j)}=p^{r(j)} e_{j}$ where $p \nmid e_{j}$. Then $r(j)>r(j+1)$ and $b_{i}=p^{r(j)} e_{i}$ for $i(j) \leqq i<$ $i(j+1)$ where $p \nmid e_{i}$. The argument of $[6$, Lemmas 8,10$]$ determines the $\bmod p$ Bockstein spectral sequence via the following

Lемма 5.3. The differential $d_{r}$ for ${ }_{p} E_{r}\left(P V_{2 n, k}\right)$ is trivial unless $r=$ $r(j) . \quad d_{r}\left(\bar{v}_{4 i(j)-1}\right)=0 \quad$ for $\quad r<r(j) . \quad d_{r(j)}\left(\bar{v}_{4 i(j)-1} \tilde{\omega}^{s}\right)=k_{j} \tilde{\omega}^{2 i(j)+s} \neq 0$ for $0 \leqq s<2[i(j+1)-i(j)], k_{j} \in Z_{p}$. If $n=2 s+1=2 N(p)+1$, $s=i(t)$ and $d_{r(t)}\left(\bar{v}_{4 s-1}\right)=k_{s} \tilde{\omega}^{2 s}$ with $k_{s} \neq 0$ and $d_{r(t)}\left(\bar{v}_{4 s-1} \tilde{\omega}\right)=0$. If $n=2 s=2 N(p)$, then $i(t)=s-1$ and $d_{r(t)}\left(\bar{v}_{4 s-5}\right)=k_{t} \tilde{\omega}^{2(s-1)} \neq 0$ and $d_{r(t)}\left(\bar{v}_{4 s-5} \tilde{\omega}^{2}\right)=0$. If $2 N(p)>n, d_{r(t)}\left(\bar{v}_{4 i(t)-1} \tilde{\omega}\right) \neq 0$ for $s<n$ $-2 i(t)$. If $2 N(p)<n-1, d_{r(t)}\left(\bar{v}_{4 i(t)-1}\right)=0$. Further, $H^{*}\left(P V_{2 n, k}\right) l$ Tors. $\otimes Z_{p}=H^{*}\left(P V_{2 n, k} ; Q\right)$ as algebras over $Z_{p}$.

We apply Poincaré duality to specify generators for $H^{*}\left(P V_{2 n, k}\right)$. Let $U$ denote the fundamental cohomology class for the closed orientable manifold $P V_{2 n, k}$ of dimension $\frac{1}{2} k(4 n-k-1)-1$. Fix an arbitrary choice of generators for $H^{*}\left(P V_{2 n, k} ; Z_{2}\right)$ such that $z_{2 s}=$ $\mathrm{Sq}^{\mathrm{l}} z_{2 s-1}$ for $s \in J_{n, k}$. Analogous to Case I, we define 2-torsion classes

$$
V_{2 s}=\delta\left(z_{2 s-1}\right) \quad \text { and } \quad U_{s, t}=\delta\left(z_{2 s-1} z_{2 t-1}\right)
$$

for $s, t \in J_{n, k}-\{N\}$ with $s<t$.

Suppose $N=l$. Note that $\pi^{*} \beta^{2}=2 e_{2 l}$ where $\rho_{2}\left(e_{2 l}\right)=z_{2 l}$. Also $\rho_{2}\left(X_{4 j-1}\right)=z_{2 j-1} z_{2 j}+z_{4 j-1}+\gamma_{j}$ for some $\gamma_{j}$ with $i^{*} \gamma_{j}=0$. So

$$
U=\omega^{l-1} e_{2 l} X_{2 n-1} \prod_{l<j<n} X_{4 j-1}
$$

since $\boldsymbol{\rho}_{p} U \neq 0$ for all primes $p$. Thus we obtain

Proposition 5.5. For $2 l<n$ with $N=l$ and $k$ odd,

$$
\begin{aligned}
H^{*}\left(P V_{2 n, k}\right) / \text { Tor }= & Z[\omega] /\left(\omega^{l}\right) \\
& \otimes Z E\left(e_{2 l}, X_{2 n-1}, X_{4 j-1} \mid l<j<n\right) .
\end{aligned}
$$

If $2 l \leqq N \leqq n$, then $N=2^{r}$ for some integer $r$. Set $s=2^{r-1}$ and note

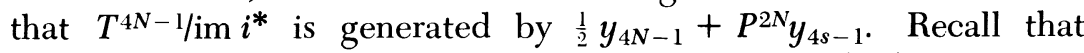
$y_{4 N-1}$ was redefined to be twice the generator in (2.3). Let $I_{2}=$ $\left\{j|2 l<2 j<n, 2| a_{j}\right\} U\{l\}$, and arrange $I_{2}$ so that

$$
l=i(0)<i(1)<\cdots<i(t) .
$$


Note that $i(t)=s$. Write $b_{i(j)}=2^{r(j)} g_{j}$ where $2 \chi g_{j}$. There exist classes $Z_{4 j-1}=z_{2 j-1} z_{2 j}+z_{4 j-1}+\gamma_{j}$ with $i^{*} \gamma_{j}=0$ for $j \in J_{n, k}-\{N\}$ such that in the $\bmod 2$ Bockstein spectral sequence we have $d_{r(j)}\left(Z_{4 i(j)-1}\right)=\alpha^{2 i(j)} \neq 0$. Any choice of the classes $X_{4 j-1}$ for $l<j<n$ with $j$ not in $I_{2}$ satisfies $\rho_{2}\left(X_{4 j-1}\right)=Z_{4 j-1}+\mu_{j}$ for some $\mu_{j}$ with $i^{*} \mu_{j}=0$. Classes $X_{4 i(j)-1}$ can be chosen so that

$$
\begin{aligned}
& i^{*} X_{4 i(j)-1}=a_{i(j)} y_{4 i(j)-1}, \\
& \text { for } 0<j \leqq t \text {. } \\
& \rho_{2}\left(X_{4 i(j)-1}\right)=\alpha^{2 i(j)-2 i(j-1)} Z_{4 i(j-1)-1},
\end{aligned}
$$

Also $\rho_{2}\left(X_{2 n-1}\right)=z_{2 n-1}+\gamma$ and $\rho_{2}\left(X_{4 N-1}\right)=z_{2 N} Z_{4 s-1}+z_{4 N-1}+\mu$ with $i^{*} \gamma=i^{*} \mu=0$ for $2 l \leqq N<n$. If $N=n, \rho_{2}\left(X_{2 n-1}\right)=z_{2 s-1} z_{2 s}$ for some choice of $X_{2 n-1}$. Thus

$$
U=\omega^{2 l-1} X_{2 n-1} \prod_{l<j<n} X_{4 j-1}
$$

since $\rho_{p}(U) \neq 0$ for all primes $p$, and we obtain the following

Proposition 5.8.

$$
H^{*}\left(P V_{2 n, k}\right) / \text { Tors }=Z[\omega] /\left(\omega^{2 l-1}\right) \otimes Z E\left(X_{2 n-1}, X_{4 j-1} \mid l<j<n\right)
$$

for $2 l \leqq N \leqq n$ with $k$ odd .

Finally we consider the case $l<N<2 l<n$ where divisibility by 2 occurs among certain products in $H^{*}\left(P V_{2 n, k}\right) /$ Tors. Note that the free class $\pi^{*} \beta^{N}=2 e_{2 N}$ with $i^{*} e_{2 N}=P^{2 N}$ and $\rho_{2}\left(e_{2 N}\right)=z_{2 N}$. Suppose $i(t)<N$ in (5.6). The higher order $\bmod 2$ Bocksteins are given by $d_{r(s)+1}\left(Z_{4 i(s)-1}\right)=z_{2 N} \alpha^{2 i(s)-N}$ for $0 \leqq s \leqq t$. Thus $X_{4 i(j)-1}$ for $1 \leqq j \leqq t$ can again be chosen to satisfy (5.7). For proper choices $\rho_{2}\left(X_{4 N-1}\right)=$ $\alpha^{2[N-i(t)]} Z_{4 i(t)-1}$ and $\rho_{2}\left(X_{2 n-1}\right)=z_{2 n-1}+\mu$ for some $\mu$ with $i^{*} \mu=0$. Now

$$
U=e_{2 N} X_{2 n-1} \omega^{2 l-N-1} \prod_{l<j<n} X_{4 j-1}
$$

since $\rho_{p}(U) \neq 0$ for all primes $p$. Note that $P^{2 N} y_{4 j-1}$ for $j \in J_{n, k}-$ $\{N\}$ survives in the integral spectral sequence for $\pi$ for $l<N<2 l<n$ so there exist classes $Y_{j}$ in $H^{*}\left(P V_{2 n, k}\right)$ for $1 \leqq j \leqq t$ such that

$$
\begin{gathered}
i^{*} Y_{j}=P^{2 N} y_{4 i(j)-1} \text { and } \\
Y_{j} \omega^{2[i(j)-i(j-1)]}=e_{2 N} X_{4 i(j)-1} \text { modulo torsion. }
\end{gathered}
$$

If $N=i(t), Y_{t}$ is not defined and $X_{4 N-1}$ can be chosen so that 
$\rho_{2}\left(X_{4 N-1}\right)=\alpha^{2[N-i(t-1)]} Z_{4 i(t-1)-1}$. In summary, we have the following

Proposition 5.10. For $l<N<2 l<n$ and $k$ odd, $H^{*}\left(P V_{2 n, k}\right) /$ Tors is generated by $\omega, \mathrm{e}_{2 N}, X_{2 n-1}, X_{4 j-1}$, and $Y_{r}$ for $l<j<n$ and $1 \leqq r \leqq t$ with $Y_{t}$ omitted if $i(t)=N$.

TheOREM 5.11. Suppose that $2 l<n$ with $k$ odd. $H^{*}\left(P V_{2 n, k}\right)$ is generated by the classes $\omega, e_{2 N}, X_{2 n-1}, X_{4 j-1}, Y_{r}, V_{2 s}$, and $U_{s, t}$ for $l<j<n$ and $s, t \in J_{n, k}-\{N\}$ with $s<t . H^{*}\left(P V_{2 n, k}\right) / T$ Tors is given by (5.5), (5.8), and (5.10).

Remark. The known result that the real geometric dimension of $m$ based on $C P^{j}$ must be greater than $j-2$ follows from the fact that $\pi^{*} \beta^{2 l}$ is in Tor $H^{*}\left(P V_{2 n, k}\right)$.

\section{REFERENCES}

1. A. Borel, Sur la cohomologie des espaces fibrés principaux et des espaces homogènes des groupes de Lie compacts, Ann. of Math. (2) 57 (1953), 115-207. MR 14, 490.

2. A. Borel and F. Hirzebruch, Characteristic classes and homogeneous spaces. II, Amer. J. Math. 81 (1959), 315-382. MR 22 \#988.

3. S. Gitler and D. Handel, The projective Stiefel manifolds. I, Topology 7 (1968), 39-46. MR 36 \#3373a.

4. S. Gitler, The projective Stiefel manifolds. II. Applications, Topology 7 (1968), 47-53. MR 36 \#3373b.

5. C. Miller, The topology of rotation groups, Ann. of Math. (2) 57 (1953), 90-114. MR 14, 673.

6. C. Ruiz, The cohomology of the complex projective Stiefel manifold, Trans. Amer. Math. Soc. 146 (1969), 541-547. MR 40 \#3584.

7. B. Steer, Une interprétation géométrique des nombres de Radon-Hurwitz, Ann. Inst. Fourier (Grenoble) 17 (1967), 209-218. MR 37 \#3590.

University of Notre Dame, Notre Dame, Indiana 46556 